\title{
Comparison of Fracture Resistance of Two Resin-based Sealers to Root Canal Walls: An In Vitro Study
}

\author{
Rashmi Issar ${ }^{1}$, Shashi Ranjan², Shashank Saurav ${ }^{3}$, Suman Kar ${ }^{4}$, Swarupananda Bera $^{5}$, Pankaj Singh ${ }^{6}$
}

\begin{abstract}
Aim and objective: In the present study, the prepared roots obturated by gutta-percha/AH plus and Resilon/Epiphany were tested and compared for fracture resistance. The study also does a scanning electron microscope (SEM) evaluation of the adaptability of these obturating materials to root canal walls.

Materials and methods: One hundred extracted mandibular premolars were decoronated and the dimensions of the roots were standardized. Each root was prepared to a size of \#25 with $6 \%$ taper. Roots were gauged after preparation and those requiring more preparation were discarded. Seventy-seven prepared roots were finally selected for the study. The samples were then divided into three groups. Group I with 25 specimens was control group in which no obturation was performed, group II with 26 specimens was obturated by gutta-percha/AH plus sealer, and group III with 26 specimens was filled by Resilon/Epiphany. The method for obturation was cold lateral condensation. The samples were then stored at $100 \%$ humidity for 2 weeks. One random sample from groups II and III was subjected to SEM analysis. Groups I, II, and III were then subjected to vertical loading in Instron machine. One-way analysis of variance (ANOVA) test and Tukey's multiple comparison test were used for statistical analysis. Results: Group III exhibited the maximum fracture resistance as compared to groups I and II. The least mean fracture resistance of $370.05 \mathrm{~N}$ was seen in group II and the maximum mean fracture resistance of $481.05 \mathrm{kN}$ was observed in group III. One-way ANOVA and Tukey's multiple comparison test between group II II, and III, group III showed a highly significant resistance to fracture as compared to groups I and II ( $p<0.0001$ ). Scanning electron microscope microphotographs showed a better adaptation of Resilon/Epiphany as compared to gutta-percha/AH plus to the root canal.
\end{abstract}

Conclusion: The Resilon/Epiphany on obturation of root canals creates a monoblock by penetrating inside the dentinal irregularities, which strengthens the root and provides fracture resistance. This fracture resistance was significantly higher in the present study as compared to groups I and II.

Clinical significance: In the present study, Resilon/Epiphany when used to obturate the prepared canals showed a promising result both in terms of fracture resistance and adaptability to root canal walls. This paves a way for the use of this combination of obturating material not only to strengthen the compromised root strength in clinical scenario but also providing an increased sealing ability which will contribute to the success of root canal treatment.

Keywords: AH plus, Epiphany, Fracture resistance, Instron, Resilon, Scanning electron microscope.

The Journal of Contemporary Dental Practice (2020): 10.5005/jp-journals-10024-2963

\section{INTRODUCTION}

Endodontology has leaped manifold in terms of success, demand, and technology. This progress has put a tremendous responsibility on the clinician to provide long-term prognosis to root canal treated tooth. Saving the tooth has become the motto of present dental sciences. Even tooth with a guarded prognosis is saved with the help of reinforcing postendodontic materials. Still the basic steps have to be followed in order to eliminate microorganisms and infected dentin during root canal treatment. Gutmann in his studies showed that removal of either coronal or radicular dentin compromises the strength of the tooth to withstand the masticatory load. In a study by Fuss et al., $10.9 \%$ of root canal treated teeth showed vertical root fracture. Endodontically treated teeth fracture because they are significantly weakened when the root canals are prepared. Due to cumulative loss of tooth structure from caries, trauma, restorative and endodontic procedures susceptibility to root fracture increases. The loss of proprioception and an elevated pain threshold allow larger loads on endodontically treated teeth without triggering a protective response. $^{1-3}$

Obturation of prepared root canals has to be performed with great proficiency to achieve desired success. Lateral condensation technique for obturation involves load application through spreaders and pluggers to the root canal wall and radicular
1,3,6 Department of Conservative Dentistry and Endodontics, Patna Dental College and Hospital, Patna, Bihar, India

${ }^{2}$ Department of Oral Pathology, Dr BR Ambedkar Institute of Dental Sciences and Hospital, Patna, Bihar, India

${ }^{4,5}$ Department of Conservative Dentistry and Endodontics, Awadh Dental College and Hospital, Jamshedpur, Jharkhand, India

Corresponding Author: Rashmi Issar, Department of Conservative Dentistry and Endodontics, Patna Dental College and Hospital, Patna, Bihar, India, Phone: +91 9835279843, e-mail: drrashmiissar@gmail. com

How to cite this article: Issar R, Ranjan S, Saurav S, et al. Comparison of Fracture Resistance of Two Resin-based Sealers to Root Canal Walls: An In Vitro Study. J Contemp Dent Pract 2020;21(11):1253-1257.

Source of support: Nil

Conflict of interest: None

dentine. In studies by Tamse et al., and Telli et al., these lateral and wedging forces of the spreader further contributes to the potential for vertical root fracture. ${ }^{4-6}$

A vital determinant for the success of endodontic treatment is the material chosen for obturation. Gutta-percha is gold standard to obturate root canals. It has several desirable properties like 
biocompatibility, chemical stability, no porosity, radiopacity, ease of manipulation, and can be removed when necessary. Despite apparently satisfactory performance over many decades, it does not reach universal idealism as it does not provide a fluid-tight seal because there is no chemical bond between gutta-percha, intraradicular dentin, and sealer. Despite development of various resin-based sealer, gutta-percha still lacks of a union between the polyisoprene component of gutta-percha and methacrylate-based resin sealers. This major disadvantage of gutta-percha has led to the need for a better product. A new promising material that rides over these flaws was desired. ${ }^{7}$

In this quest for bonding to the dentinal walls of the root canal, Resilon/Epiphany was developed. According to Williams et al., this material not only provides a good seal but also reinforces the root canal treated tooth. Resilon is a thermoplastic synthetic polymerbased material which has properties desirable for root canal filling, adhesion to dentin, good sealing ability that looks and handles like gutta-percha. Epiphany is a methacrylate resin-based sealer and has its main constituents as bisphenol-A diglycidyldimethacrylate (BisGMA), other resins, and fillers. ${ }^{8-10}$

This system also uses a primer to prepare the tooth root surface by etching, which creates finger-like dentinal projections facilitating a lock and key type of bonded seal between the tooth, sealer, and core material. Teixeira et al., reported that when the canals are filled with Resilon/Epiphany, it formed a monoblock within the canals that bonds to the dentinal walls. Because the resin core, sealant, and dentinal wall all are "attached", it appears logical that they have the potential to strengthen the root against fracture. ${ }^{9}$

This study was undertaken to determine the fracture resistance of roots obturated with gutta-percha/AH plus sealer and Resilon/ Epiphany by cold lateral condensation technique and scanning electron microscope (SEM) evaluation was performed to analyze the adaptation of obturating materials to root canals.

\section{Materials and Methods}

One hundred extracted mandibular single-rooted premolars were collected from the Department of Oral and Maxillofacial Surgery, Patna Dental College and Hospital. The teeth were extracted for orthodontic reasons.

Inclusion criteria were single-rooted mandibular premolars with minimum root length of $14 \mathrm{~mm}$, maximum mesiodistal dimension of 4-5 mm, and buccolingual diameter of 8-9 mm. Exclusion criteria for teeth selection were root caries, preexisting root fracture, open apex, calcified canals, roots with resorptive defects, curved roots, and roots which had to be prepared more than \#25,6\% taper.

Teeth were decoronated with the help of a diamond disk mounted on a DFS mandrel in a straight handpiece at 30,000 rpm (Fig. 1A). Each root was measured and selected according to inclusion criteria. The measurements were performed by Vernier Calliper (Fig. 1B). Roots with preexisting root fracture were ruled out under $\times 10$ magnification. Patency of the canals was checked and the glidepath established. After determination of working length, each tooth was prepared by Neo Endo flex rotary files by using a 6:1 reduction handpiece (X-SMART plus; Dentsply Maillefer) at a speed of $250 \mathrm{rpm}$. All the canals were prepared up to $\# 25,6 \%$. A \#25 hand K-file of $2 \%$ taper was used to gauge the size of apical foramen. If the root canal needed preparation more than \#25,6\% it was not included in the final study sample. A total of 77 samples were finally selected for the study. Irrigation was performed by $3 \%$ sodium hypochlorite (Septodont) and a final rinse by $17 \%$ ethylene diamine tetraacetic acid (EDTA) (PrevestDenPro) for 1 minute. All specimens were lastly irrigated with normal saline and dried by paper points. Teeth were assigned randomly into three groups.

- Group I (25 samples): control group-no obturation was performed in this group.

- Group II (26 samples): obturated by gutta-percha/AH plus sealer with cold lateral condensation (Fig. 1C).

- Group III (26 samples): obturated by Resilon/Epiphany with cold lateral condensation (Fig. 1D).

The root orifices of all specimens were sealed by Cavit G (ESPE, $3 \mathrm{M}$, St. Paul, MN). Root specimens from each group were stored at $37^{\circ} \mathrm{C}$ and $100 \%$ humidity for 2 weeks. One sample each from groups II and III was longitudinally sectioned by indenting with a diamond disc and splitting by spatula and was evaluated using SEM (Fig. 1E) at coronal, middle, and apical third for adaptation to root canal walls (Bose Institute, Kolkata).

Apical root end for remaining samples from all three groups was then embedded in a polyvinyl ring, leaving $9 \mathrm{~mm}$ of the root exposed, and $5 \mathrm{~mm}$ embedded. Acrylic resin was poured into polyvinyl ring, on a stable and smooth base. Each root was mounted when the acrylic resin was in dough stage.

Mechanical testing for fracture resistance was performed by Instron machine (NIIT Patna, Physics Dept.) for all the three groups (Fig. 1F). The loading fixture had a spherical tip of radius $2 \mathrm{~mm}$. Application of a vertical loading force of $1.00 \mathrm{~mm}$ per minute was performed till the sample fractured. The force exerted at the time of vertical fracture of the sample was recorded in kilo Newton $(\mathrm{kN})$ on the dial gauge of the Universal testing Instron machine. Samples of all the three groups underwent this fracture analysis and the values were recorded individually. The values obtained for fracture resistance were recorded and mean calculated for each group for statistical analysis.

\section{Statistical Analysis}

One-way analysis of variance (ANOVA) test and Tukey's multiple comparison test were used for statistical analysis.

\section{Results}

The mean values of fracture resistance calculated after taking the average from 25 samples in each group was-Group I-446.19 kN, Group II-370.05 kN, and Group III-480.05 kN. The mean values showed that fracture resistance was maximum with group III and minimum with group II in comparison to group I (control group). One-way ANOVA revealed highly significant difference between groups I, II, and III ( $p<0.001$ ). Roots obturated by Resilon/Epiphany (group III) had the highest mean fracture resistance of 480.05 $\mathrm{kN}$ on subjecting to vertical loading forces by Universal Instron machine. The mean values for group III were statistically significant as compared to groups II and III when subjected to Tukey's multiple comparison test. Roots obturated by gutta-percha group (group II) displayed least mean fracture resistance of $370.05 \mathrm{kN}$ (Table 1 and Fig. 2).

One sample each was analyzed for adaptability of the root canal filling material to root canal dentin under SEM from groups II and III. Scanning electron microscope findings of the adaptation of gutta-percha/AH plus sealer to the canal walls showed gaps 

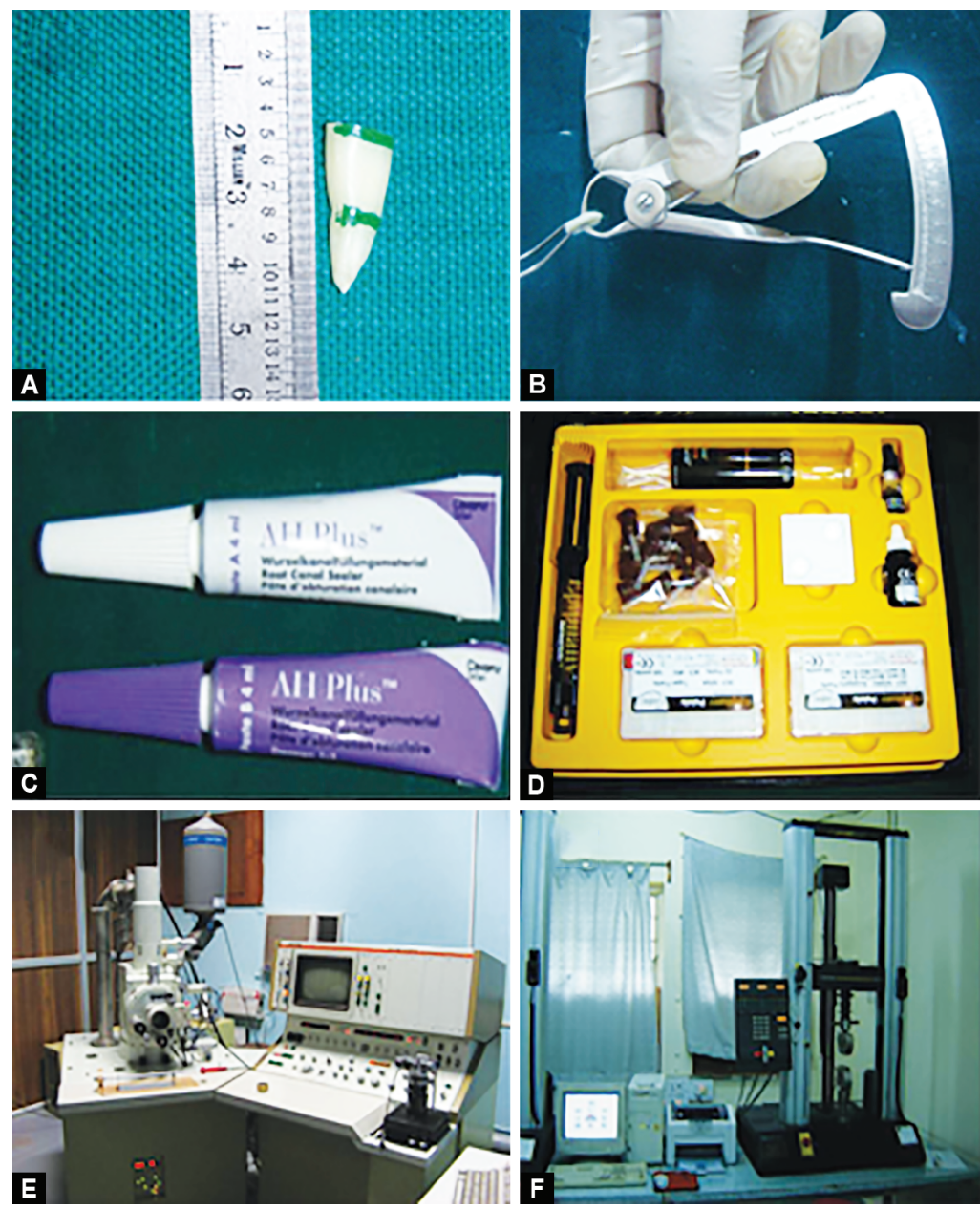

Figs $1 \mathrm{~A}$ to F: (A) Decoronated root marked at $5 \mathrm{~mm}$ to be embedded in acrylic; (B) Vernier caliper measuring root length and width; (C) AH plus sealer; (D) Resilon/Epiphany kit; (E) SEM equipment; (F) Instron machine

Table 1: Mean forces required to fracture prepared roots

\begin{tabular}{llllll}
\hline Group & No. of samples & Mean yield load $(k N)$ & SD & Min. & Max. \\
\hline I & 25 & 446.19 & 7.99 & 434.69 & 456.41 \\
II & 25 & 370.05 & 3.73 & 364.76 & 375.86 \\
III & 25 & 481.05 & 5.97 & 469.18 & 494.14 \\
\hline
\end{tabular}

at all the three levels, i.e., coronal, middle, and apical third (Figs 3A, C and E). While SEM photographs at 500 $\times$ magnification showed no gaps and close adaptation for obturated canals by Resilon/Epiphany (Figs 3B, D and F). No SEM analysis for control group was performed.

The results in the study showed the highest mean fracture resistance in Resilon/Epiphany group which on subjecting to one-way ANOVA and Tukey's multiple comparison test to other groups showed a highly significant result $(p<0.0001)$. Even the adaptation to root canal walls of group III was more intimate to the root canal walls than group II at all the three levels.

\section{Discussion}

The fracture resistance of an endodontically treated tooth reduces due to loss of tooth structure, instrumentation, decrease in modulus of elasticity caused by dentin dehydration by irrigants, and forces applied during root canal obturation. ${ }^{2}$

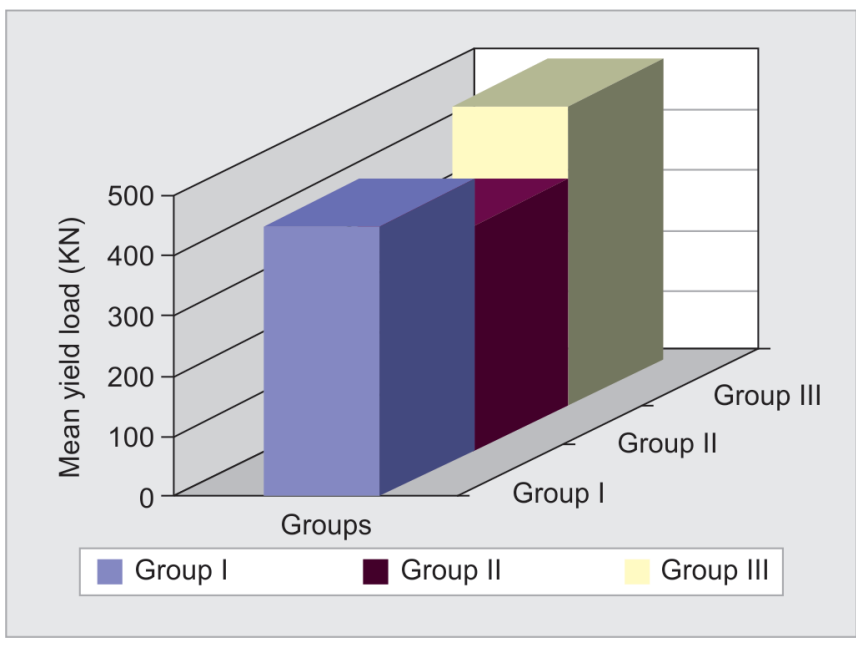

Fig. 2: Comparison of fracture resistance of groups I, II, and III 

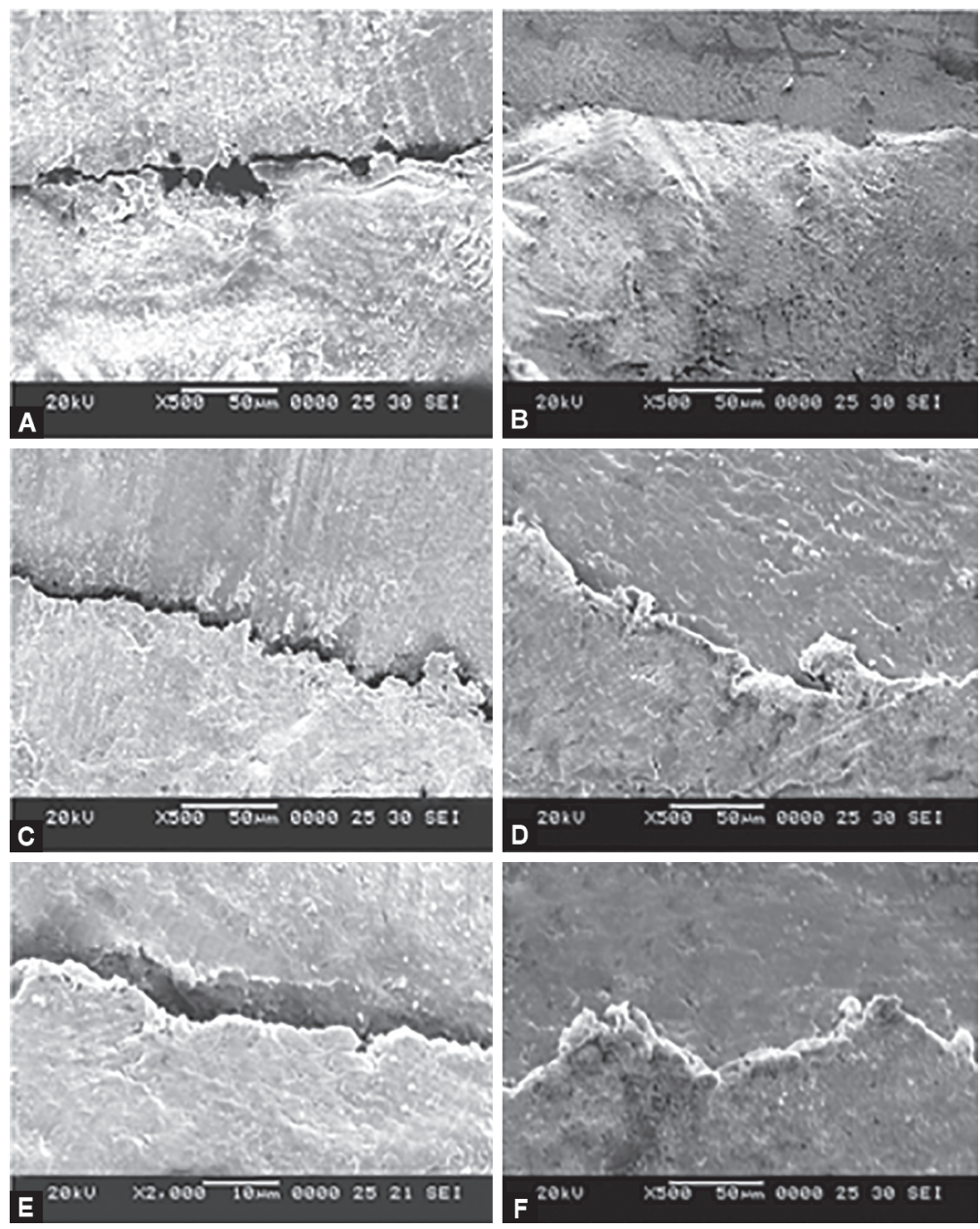

Figs $3 A$ to F: SEM microphotographs comparing adaptation of obturating materials to canal $(\times 500)$ : (A) At coronal third GP/AH plus; (B) At coronal third Resilon/Epiphany; (C) At middle third GP/AH plus; (D) At middle third Resilon/Epiphany; (E) At apical third GP/AH plus; (F) At apical third Resilon/Epiphany

The present study compared the fracture resistance of unobturated roots to those obturated by gutta-percha/AH plus and Resilon/Epiphany.

The mesiodistal and buccolingual width and length of root canals must be standardized for the extracted tooth. In the present study, all roots were standardized in terms of size of preparation and root dimensions. Rotary, NeoEndo flex files have been used for root canal preparation which results in a round cross-section and equal distribution of stress in root during filling, also risk of root fracture decreases.

Irrigation of the canals was performed using 3\% sodium hypochlorite and last rinse of EDTA to remove the smear layer. Due to low surface tension, EDTA easily penetrates into the dentinal tubules and dissolves the smear layer as deep as $2.5-4 \mu \mathrm{m}$ which increases the bond and adaptation of sealer to root canal walls. Ethylene diamine tetraacetic acid if in contact with dentin for longer duration leads to erosion of dentin, so an EDTA rinse of only 1 minute was used in the present study. Lastly, distilled water was used to neutralize and wash out the remaining irrigating solutions. ${ }^{11,12}$

The most widely used method of obturation, i.e., cold lateral condensation technique was used in the present study. Shashidhar et al., compared the fracture strength of teeth filled with lateral and vertical condensation techniques. The maximum fracture resistance of teeth was obtained after filling by the lateral condensation technique. In the present study, the unobturated canals (group I) showed a higher fracture resistance than group II which clearly shows that the forces applied during lateral condensation compromises with the fracture resistance of tooth. ${ }^{13}$

In the present study using a universal testing machine, the load was vertically applied on the longitudinal axis of the root to transfer the load entirely to the root. The individual fracture resistance was recorded and mean was calculated for groups I, II, and III. The values obtained were subjected to Dunnett $D$ test and Tukey's multiple comparison test for statistical analysis. The canals obturated by gutta-percha/AH plus had least fracture resistance as compared to those obturated by Resilon/Epiphany and control group. While the highest mean fracture resistance was shown by Resilon/Epiphany group (Table 1 and Fig. 2).

In a study, Kumar et al., compared the fracture resistance of Resilon/Realseal and gutta-percha/AH plus obturated by thermoplasticed obturation technique. They found that forces at fracture were significantly higher in Resilon group as compared to the gutta-percha group, similar to the present study. ${ }^{14}$

Teixeira et al., in his study compared a resin-based obturating material and gutta-percha for fracture resistance. They concluded that teeth obturated with resin-based system had higher fracture resistance than the gutta-percha group. The results obtained were statistically insignificant. The results in the present study were 
statistically significant as compared to the study by Teixeira et al., which could be due to difference in method of obturation. ${ }^{9}$

Ashraf et al., in their study used roots of maxillary incisors and RACE rotary files for preparation of root canal and obturated with the same materials as used in the present study. The results were in agreement with the present study as were the results in studies conducted by Monteiro et al., and Ahlberg and Tay., ${ }^{9,15-17}$

Contradicting the results of the present study, Sagsen et al., compared the fracture resistance of the root-filled teeth with Resilon/Epiphany sealer, gutta-percha/AH-26, and showed no statistically significant differences between the two groups. Topcuoğlu et al., reported no significant difference in root dentin fracture resistance of root canals filled with gutta-percha/AH-plus or gutta-percha/Endosequence BC sealer. The difference could be attributed to the selection criteria of roots, different loads applied during testing, and different storing condition of roots. ${ }^{18,19}$

On SEM analysis of one specimen each from groups II and III, a better adaptation of Resilon/Epiphany was observed in comparison to gutta-percha/AH plus in coronal, middle, and apical third but further studies with more samples are needed to validate it (Fig. 3). ${ }^{20,21}$

The limitation of the study was no comparison with uninstrumented root samples, no analysis of microtensile bond strength, usage of only one method of obturation, and not taking into consideration other variables that might affect the root strength like irrigating and chelating agents used during root canal preparation.

\section{CONCLUSION}

In the present study, the highest mean fracture resistance was shown in the root canals obturated by Resilon/Epiphany and the result was highly significant ( $p<0.0001)$ as compared to other groups. Also, this group in SEM microphotographs showed better adaptation to root canal walls in coronal, middle, and apical third. In future, Resilon/Epiphany can substitute gutta-percha as an obturating material showing a great potential in terms of strengthening root canal walls and resistance to microleakage through better adaptation as seen in SEM photographs. Although other variables like microleakage analysis and long-term in vivo follow-up studies are needed in to establish its clinical application.

\section{References}

1. KIvanc BH, Alacam T, Ulusoy OIA, et al. Fracture resistance of thinwalled roots restored with different post systems. Int Endod J 2009;42(11):997-1003. DOI: 10.1111/j.1365-2591.2009.01609.x.

2. Sedley MC, Messer H. Are endodontically teeth more brittle? J Endod 1992;18(7):332-335. DOI: 10.1016/S0099-2399(06)80483-8.

3. Fuss Z, Lustig J, Tamse A. Prevalence of vertical root fractures in extracted endodontically treated teeth. J Endod 1999;32:283-286. DOI: 10.1046/j.1365-2591.1999.00208.x.
4. Tamse A, Lustig J, Kaplavi J. An evaluation of endodontically treated vertically fractured teeth. J Endod 1999;25(7):506-508. DOI: 10.1016/ S0099-2399(99)80292-1.

5. Telli C, Gulkan P, Raab W. Additional studies on the distribution of stress during vertical compaction of gutta-percha in root canal. $\mathrm{Br}$ Dent J 2007;187(1):1-7.

6. Lertchirakarn V, Palamara J, Masser H. Load and strain during lateral condensation and vertical fracture. J Endod 1999;25(2):99-104. DOI: 10.1016/S0099-2399(99)80005-3.

7. Gatewood RS. Endodontic materials. Dent Clin North Am 2007;51(3):695-712. DOI: 10.1016/j.cden.2007.04.005.

8. Williams C, Loushine RJ, Weller NR, et al. A comparison of cohesive strength and stiffness of Resilon and gutta-percha. J Endod 2006;32(6):553-555. DOI: 10.1016/j.joen.2005.08.002.

9. Teixeira FB, Teixeira EC, Thompson JY, et al. Fracture resistance of roots endodontically treated with a new resin filling material. J Am Dent Assoc 2004;135(5):646-652. DOI: 10.14219/jada.archive.2004.0255.

10. Barnett $F$, Trope $M$. Resilon ${ }^{\mathrm{TM}}$ : a novel material to replace gutta-percha. Oral Health 2004.

11. Calt S, Serper A.Time-dependent effects of EDTA on dentin structures. J Endod 2002;28(1):17-19. DOI: 10.1097/00004770-200201000-00004.

12. Sim $\mathrm{T}$, Knowles J, Ng YL, et al. Effect of sodium hypochlorite on mechanical properties of dentine and tooth surface strain. Int Endod J 2001;34(2):120-132. DOI: 10.1046/j.1365-2591.2001.00357.x.

13. Shashidhar C, Shivanna V, Shivamurthy G, et al. The comparison of microbial leakage in roots filled with resilon and gutta-percha: an in vitro study. J Conserv Dent 2011;14(1):21-27. DOI: 10.4103/09720707.80725.

14. Kumar P, Kaur NM, Arora S, et al. Evaluation of fracture resistance of roots obturated with resilon and thermoplasticized gutta-percha: an in vitro study. J Conserv Dent 2014;17(4):354. DOI: 10.4103/09720707.136510 .

15. Ashraf H, Momeni G, Majd NM, et al. Fracture resistance of root canals obturated with gutta-percha vs Resilon with two different techniques. Iran Endod J 2013;8(3):136-139.

16. Monteiro J, de Ataide IdeN, Chalakkal $\mathrm{P}$, et al. In vitro resistance to fracture of roots obturated with Resilon or guttapercha. J Endod 2011;37(6):828-831. DOI: 10.1016/j.joen.2011.02.024.

17. Ahlberg K, Tay WM. A methacrylate based cement used as a rootcanal sealer. Int Endod J 1998;31(1):15-21. DOI: 10.1046/j.1365-2591.1998. t01-1-00118.x.

18. Sagsen B, Er O, Kahraman $Y$, et al. Resistance to fracture of roots filled with three different techniques. Int Endod J 2007;40(1):31-35. DOI: 10.1111/j.1365-2591.2006.01177.x.

19. Topcuoğlu HS, Tuncay O, Karataş E, et al. In vitro fracture resistance of roots obturated with epoxy resin-based, mineral trioxide aggregatebased, and bioceramic root canal sealers. J Endod 2013;39(12):16301633. DOI: 10.1016/j.joen.2013.07.034.

20. Patri G, Agrawal P, Anushree N, et al. A scanning electron microscope analysis of sealing potential and marginal adaptation of different root canal sealers to dentin - an in vitro study. J Contemp Dent Pract 2020;21(1):73-77. DOI: 10.5005/jp-journals-10024-2733.

21. Rouhani A, Ghodussi J, Naghavi N, et al. Scanning electron microscope evaluation of dentinal tubule penetration of Epiphany in severely curved root canals. Eur J Dent 2013;7(4):423-428. DOI: 10.4103/13057456.120673 . 